Pacific Journal of Mathematics

PLANO MODELS WITH MANY GENERIC CLASSES 


\title{
PEANO MODELS WITH MANY GENERIC CLASSES
}

\author{
JAMES H. SCHMERL
}

The famous theorem of MacDowell and Specker asserts that every model of Peano arithmetic has a proper elementary end-extension. A consequence of their theorem (and its proof) is that every model of Peano arithmetic of cardinality less than $\kappa$ has a $\kappa$-like elementary end-extension, and, in addition, if $\kappa$ is regular, then there is such a $\kappa$-like model in which all classes are definable. However, under the assumption of the existence of a $\kappa$-Kurepa tree, each model of Peano arithmetic of cardinality less than $\kappa$ does have a $\kappa$-like elementary end-extension in which there are more than $\kappa$ generic classes.

Introduction. We assume that we have some fixed countable similarity type which includes symbols denoting the usual arithmetic operations and relations: $+, \cdot,<, 0,1$. Then Peano arithmetic, denoted by $\boldsymbol{P}$, is the theory which, besides the sentences describing the trivial arithmetic properties, includes all the instances of the induction scheme. Thus, if $\varphi\left(x_{0}, \cdots, x_{n-1}, y\right)$ is an $(n+1)$-ary formula, then the universal closure of

$$
[\varphi(\bar{x}, 0) \wedge \forall y(\varphi(\bar{x}, \mathrm{y}) \longrightarrow \varphi(x, y+1))] \longrightarrow \forall y \varphi(\bar{x}, y)
$$

is in $P$.

Henceforth, all models considered are models of Peano arithmetic. We denote these models by $\mathscr{N}$ and $\mathscr{C}$ with universes of $N$ and $M$ respectively.

For any set $X$ we denote by $X^{n}$ the set of $n$-tuples of elements of $X$. If $\bar{a} \in X^{n}$ (the bar being only for emphasis), we let $a_{i}$ be the $i$ th coordinate of $\bar{a}$. Thus if $\bar{a} \in X^{n}$, then $\bar{a}=\left\langle a_{0}, \cdots, a_{n-1}\right\rangle$. If $\bar{X}=$ $\left\langle X_{0}, \cdots, X_{n-1}\right\rangle$ we occasionally write $\bar{x} \in \bar{X}$ for $\bar{x} \in X_{0} \times \cdots \times X_{n-1}$.

For any $\mathscr{N}$ a set $R \subset N^{n}$ is definable (in $\mathscr{N}$ ) if it is defined in $\mathscr{N}$ by a formula in which parameters from $N$ are allowed. A set $X \subset N$ is $\mathscr{N}$-finite iff $X$ is bounded and definable. If $X$ is $\mathscr{N}$-finite and $R \subset N^{n+1}$ is definable, then $\left\{Q \subset N^{n}:\{x\} \times Q \subset R\right.$ for some $x \in X\}$ is an $\mathscr{N}$-finite collection of sets. We choose some binary formula, say $\varphi(x, y)$, which indexes finite sets. That is, for any model $\mathscr{C}$ let $F^{\mathscr{N}}$ be a function such that if $a \in M$, then $F^{\mathscr{N}}(a)=$ $\{b \in M: \mathscr{C} \vDash \varphi(a, b)\}$. Then $F^{\mathscr{N}}$ is a bijection from $M$ to the set of $\mathscr{C}$-finite sets. We define the binary relation $\triangleleft$ by $x \triangleleft y$ iff $F(x)$ is a proper initial segment of $F(y)$. Notice that $(M, \triangleleft)$ is treelike in the sense that $\triangleleft$ is a partial ordering of $M$ such that the set of 
predecessors of any element is linearly ordered. In general, $(M, \triangleleft)$ is not well-founded although every nonempty definable set has a $\triangleleft$-minimal element. If $\bar{x}=\left\langle x_{0}, \cdots, x_{n-1}\right\rangle$ and $\bar{y}=\left\langle y_{0}, \cdots, y_{n-1}\right\rangle$, we set $\bar{x} \triangleleft \bar{y}$ iff $x_{i} \triangleleft y_{i}$ for each $i<n$.

We say that $\mathscr{K}$ is an end-extension of $\mathscr{N}$ iff $\mathscr{N} \subset \mathscr{M}$ and $N$ is a <-initial segment of $M$. The model $\mathscr{N}$ is $\kappa$-like iff card $(N)=$ $\kappa$ but every $\mathscr{N}$-finite set has cardinality $<\kappa$.

In proving the MacDowell-Specker Theorem one shows that an ultrafilter can be defined in any model and that the ultrapower is then a proper elementary end-extension. Of course, the ultrafilter is not over the set of all subsets of the model but only over the Boolean algebra of the definable sets. Then in constructing the ultrapower one only considers the definable functions. This type of construction originated with Skolem [6], who used it to get nonstandard models of arithmetic.

We use a slightly more general type of construction. Suppose that $\mathscr{N}$ is a model and $U$ an ultrafilter over the Boolean algebra of all definable $n$-ary relations of $\mathscr{N}$ : Then let $\mathscr{H}$ be the ultrapower of $\mathscr{N}$ restricted to definable $n$-ary functions. It is routine to verify in such a setting that Łos' Theorem (see, for example, [1, Theorem 2.2]) still remains true; in particular, $\mathscr{N}$ is elementarily embeddable in $\mathscr{H}$ in a canonical way. We call such an $\mathscr{C}$ a Skolem ultrapower of $\mathscr{N}$, and denote it by $\mathscr{N}^{U}$.

In $\S 1$ we give a proof of the MacDowell-Specker Theorem. It is then shown that the construction results in models with few classes. In particular, if $\kappa$ is regular, then in any $\kappa$-like model constructed by their method all the classes are definable.

We discuss generic classes in $\S 2$. Whenever $c f(\kappa)>\omega$, then in any $\kappa$-like model constructed by the MacDowell-Specker method there are no generic classes. This is in contrast with Theorem 2.7 which asserts that in any model with cofinality $\omega$ there are a host of generic classes.

In $\S 3$ we show how to construct elementary end-extensions which have many classes. Theorem 3.1 extends the MacDowellSpecker Theorem. If $\mathscr{X}$ is an $\mathscr{N}$-generic collection of classes, then $(\mathscr{N} ; \mathscr{Z})$ has an elementary end-extension in which each $X \in \mathscr{Z}$ is properly extended. From this we can deduce one of the main results of this paper: If there exists a $\kappa$-Kurepa tree and if $\mathscr{N}$ is a model such that card $(N)<\kappa$, then $\mathscr{N}$ has a $\kappa$-like elementary end-extension with at least $\kappa^{+}$classes. The methods used for constructing such models owe much to similar methods developed by Keisler [3]. In fact, he constructed such models in the case $\kappa=\omega_{1}$. 
He also did so in the case that $\kappa=\lambda^{+}$, card $(N)=\lambda$, and $\mathscr{N}$ is saturated.

1. The MacDowell-Specker Theorem. In this section we sketch a proof of the MacDowell-Specker Theorem. We do this in order to show that if $\kappa$ is regular and $\mathscr{N}$ is a $\kappa$-like model constructed according to their prescription, then all classes are definable. This fact, which is Theorem 1.5, was pointed out to me by S. Simpson.

THEOREM 1.1. (MacDowell-Specker). Every model of $\boldsymbol{P}$ has a proper elementary end-extension.

Proof. Let $\left\langle\varphi_{i}(x, y): i\langle\omega\rangle\right.$ be a list of all formulas of two variables. Then, using the fact that induction holds, we can find formulas $\psi_{i}(x)$ such that for each $n<\omega$ the following is a theorem of $\boldsymbol{P}$ :

$$
(\exists y>z)(\forall x<w) \bigwedge_{i<n}\left(\varphi_{i}(x, y) \longleftrightarrow \psi_{i}(x)\right) .
$$

Let $\mathscr{N}$ be a model of $\boldsymbol{P}$ and and let

$$
U=\left\{\left\{b \in N: \mathscr{N} \vDash \varphi_{i}(a, b) \longleftrightarrow \psi_{i}(a)\right\}: a \in N \text { and } i<\omega\right\} .
$$

Then $U$ is an ultrafilter over the Boolean algebra of definable subsets of $\mathscr{N}$. Clearly $\mathscr{N}^{U}$ is a proper elementary end-extension of $\mathscr{N}$.

In the proof of Theorem 1.1 the ultrafilter $U$ and, consequently, also the extension $\mathscr{N}^{U}$ are completely determined by the sequences $\left\langle\varphi_{i}(x, y): i\langle\omega\rangle\right.$ and $\left\langle\psi_{i}(x): i\langle\omega\rangle\right.$. We call any such extension $\mathscr{N}^{U}$ of $\mathscr{N}$ a MacDowell-Specker extension of $\mathscr{N} \cdot$ A MacDowell-Specker chain is a sequence $\left\langle\mathscr{N}_{2}: \nu\langle\alpha\rangle\right.$ such that each $\mathscr{N}_{\nu+1}$ is a MacDowellSpecker extension of $\mathscr{N}_{\nu}$, and for $\delta$ a limit ordinal $\mathscr{N}_{\delta}=U\left\{\mathscr{N}_{\nu}\right.$ : $\nu<\delta\}$.

Definition 1.2. If $\mathscr{N}$ is a model of $P$ and $X \subset N$, then $X$ is a class (of $\mathscr{N}$ ) iff $X$ is not $\mathscr{N}$-finite but the intersection of $X$ with each $\mathscr{N}$-finite set is $\mathscr{N}$-finite.

Every class is unbounded, and every unbounded, definable set is a class. A class can be thought of as a branch of the treelike structure $(N, \triangleleft)$ which has the same length as $(N, \triangleleft)$. Thus $B$ is such a branch iff $\bigcup\{F(b): b \in B\}$ is a class.

Lemma 1.3. If $\mathscr{t}$ is an elementary end-extension of $\mathscr{N}^{-}$and $X$ is a class of $\mathscr{A}$, then $X \cap N$ is a class of $\mathscr{N}$. 
Proof. One need only notice that a subset of $N$ is $\mathscr{N}$-finite iff it is $\mathscr{C}$-finite.

Lemma 1.4. If $\mathscr{C}$ is a MacDowell-Specker extension of $\mathscr{N}$ and $X$ is a class of $\mathscr{M}$, then $X \cap N$ is definable in $\mathscr{N}$.

Proof. Let $X \subset M$ be a class of $\mathscr{C}$ and let $b \in X-N$. Then there is $c \in M$ such that $F(c)=\{x \in X: x<b\}$. But in the construction of $\mathscr{K}$, the element $c$ corresponds to a function definable in $\mathscr{N}$. Hence clearly $\{a \in N: a \in F(c)\}=X \cap N$ is definable in $\mathscr{N}$.

THEOREM 1.5. If $\left\langle\mathscr{N}_{\nu}: \nu \leqq \kappa\right\rangle$ is a MacDowell-Specker chain, where $\kappa$ is regular and card $\left(N_{0}\right)<\kappa$, then $\mathscr{N}_{\kappa}$ is a $\kappa$-like elementary end-extension of $\mathscr{N}_{0}$ in which every class is definable.

Proof. It is clear that $\mathscr{N}_{\kappa}$ is a $\kappa$-like elementary end-extension of $\mathscr{N}_{0}$. Now let $X$ be a class of $\mathscr{N}_{n}$. Then using the regularity of $\kappa$ there is $\nu<\kappa$ such that $\left(\mathscr{N}_{\nu}, X \cap N_{\nu}\right) \prec\left(\mathscr{N}_{\kappa}, X\right)$. By Lemma 1.3, $X \cap N_{\nu+1}$ is a class of $\mathscr{N}_{\nu+1}$; then by Lemma $1.4, X \cap N_{\nu}$ is definable in $\mathscr{N}_{\nu} \cdot$ But then $X$ is definable in $\mathscr{N}_{\kappa}$.

Let $\mathscr{X}$ be a collection of classes of $\mathscr{N}$. We denote the structure $(\mathscr{N}, X)_{X \in \mathscr{Z}}$ by $(\mathscr{N}, \mathscr{X})$. We say $\mathscr{X}$ satisfies replacement iff for each $(n+2)$-ary formula $\varphi(\bar{x}, u, v)$ in the language of $(\mathscr{N}, \mathscr{X})$ the following is true in $(\mathscr{N}, \mathscr{E})$ :

$$
\forall y \exists z(\forall u<y)[\exists v \varphi(\bar{x}, u, v) \longrightarrow(\exists v<z) \varphi(\bar{x}, u, v)] .
$$

Similarly, we say that $\mathscr{P}$ satisfies induction iff for each $(n+1)$-ary formula $\varphi(\bar{x}, y)$, the following is true in $(\mathscr{N}, \mathscr{X})$ :

$$
[\varphi(\bar{x}, 0) \wedge \forall y(\varphi(\bar{x}, y) \longrightarrow \varphi(\bar{x}, y+1))] \longrightarrow \forall y \varphi(\bar{x}, y) .
$$

We say that $X$ satisfies replacement or induction whenever $\{X\}$ does. It is well-known that $\mathscr{X}$ satisfies replacement whenever it satisfies induction. In light of the fact that for regular $\kappa$ the collection of all classes of $a \kappa$-like model satisfies replacement, the next theorem extends the previous one.

THEOREM 1.6. If $\left\langle\mathscr{N}_{\nu}: \nu \leqq \alpha\right\rangle$ is a MacDowell-Specker chain, where $\operatorname{cf}(\alpha)>\omega$, then $\mathscr{N}_{\alpha}$ is an elementary end-extension of $\mathscr{N}_{0}$ in which every class satisfying replacement is definable.

Proof. The proof is like that of the previous theorem; however, we use replacement as a substitute for regularity. Let $X$ be a class of $\mathscr{N}_{\alpha}$ which satisfies replacement. Then we need only note 
that, due to the fact that $c f(\alpha)>\omega$, there is $\nu<\alpha$ such that $\left(\mathscr{N}_{\nu}, X \cap N_{\nu}\right) \prec\left(\mathscr{N}_{\alpha}, X\right)$.

2. Generic classes. A property that a class can have is that of being generic. In this section we consider generic classes. More generally, we consider generic collections of classes.

Definition 2.1. If $R \subset N^{n}$, then $R$ is dense (in $\mathscr{N}$ ) iff for each $\bar{a} \in N^{n}$ there is $\bar{b} \in R$ such that $\bar{a} \triangleleft \bar{b}$.

Definition 2.2. Let $\mathscr{N}$ be a model of $\boldsymbol{P}$. A collection $\mathscr{X}$ of subsets of $N$ is $\mathscr{N}$-generic iff whenever $\bar{X} \in \mathscr{Z}^{n}$ and $R \subset N^{n}$ is a definable dense relation, then there is $\bar{a} \in R$ such that each $F\left(a_{i}\right)$ is an initial segment of $X_{i}$. Also, $X \subset N$ is $\mathscr{N}$-generic iff $\{X\}$ is $\mathscr{N}$ generic.

Lemma 2.3. If $\mathscr{X}$ is $\mathscr{N}$-generic, then each $X \in \mathscr{X}$ is a nondefinable class.

Proof. Let $X \in \mathscr{Z}$. Clearly $\{a \in N: F(a)$ is not an initial segment of $X\}$ is dense, so that $X$ is not definable. To show $X$ is a class, for each $b \in N$ let $Y_{b}=\{a \in N$ : there is $c \in F(a)$ for some $c>b\}$. (Think of $Y_{b}$ as the set of finite sets which have an element $>b$.) It is clear that $Y_{b}$ is a definable dense set. Thus, for any $b \in N$ there is $a \in Y_{b}$ such that $F(a)$ is an initial segment of $X$. Thus, since $\{a \in X: a \leqq b\}$ is a proper subset of $F(a)$, it follows that $X$ is a class.

LEMma 2.4. Let $\left\langle\mathscr{N}_{2}: \nu\langle\delta\rangle\right.$ be such that if $\nu<\mu<\delta$ then $\mathscr{N}_{\mu}$ is an elementary end-extension of $\mathscr{N}_{\nu} \cdot$ Let $\mathscr{N}=\bigcup\left\{\mathscr{N}_{\nu}: \nu<\delta\right\}$ and let $\mathscr{X}$ be a collection of subsets of $N$ such that whenever $\nu<\delta$, then $\left\{X \cap N_{\nu}: X \in \mathscr{X}\right\}$ is $\mathscr{N}_{\nu}$-generic. Then $\mathscr{X}$ is $\mathscr{N}$-generic.

Proof. Let $R \subset N^{n}$ be a definable dense relation, and let $\bar{X} \in \mathscr{Q}^{n}$. There is some $\nu<\delta$ such that $R \cap N_{\nu}^{n}$ is a definable dense relation in $\mathscr{N}_{\nu}$. Then there is $\bar{a} \in R \cap N_{\nu}^{n}$ such that each $F\left(a_{i}\right)$ is an initial segment of $X_{i} \cap N_{i}$. But then $\bar{a} \in R$ and each $F\left(a_{i}\right)$ is an initial segment of $X_{i}$.

The next two lemmas can be proved by forcing. We use as conditions the sets which are finite in the sense of the model, and $p$ extends $q$ when $q \triangleleft p$. Then standard forcing techniques are used. See, for example, [5].

Lemma 2.5. If $\mathscr{Z}$ is $\mathscr{N}$-generic, then $\mathscr{X}$ satisfies induction. 
Lemma 2.6. If $\mathscr{C}$ is an elementary end-extension of $\mathscr{N}$ and $\mathscr{Z}$ and $\mathscr{Y}$ are respectively $\mathscr{N}$-generic and $\mathscr{C}$-generic where $\mathscr{X}=$ $\{Y \cap N: Y \in \mathscr{Y}\}$, then $(\mathscr{N}, \mathscr{Z}) \prec(\mathscr{M}, \mathscr{Y})$.

The existence of generic classes in certain models is shown in the next theorem. For any model $\mathscr{N}$ we let $\operatorname{cf}(\mathscr{N})$ be the cofinality of the order type of $\mathscr{N}$.

THEOREM 2.7. If $\operatorname{cf}(\mathscr{N})=\omega$, then there is an $\mathscr{N}$-generic $\mathscr{X}$ such that each $\mathscr{N}$-finite set is an initial segment of some $X \in \mathscr{X}$.

Proof. The proof consists of showing that working inside of $\mathscr{N}$ we can find generic classes. For each $j\left\langle\omega\right.$ let $\left\langle\varphi_{i}^{j}(x, \bar{y}): i\langle\omega\rangle\right.$ be a list of all formulas with the $j+1$ free variables $x, y_{0}, \cdots, y_{j-1}$. For each $x \in N$, let $R_{i, x}^{j} \subset N^{j}$ be such that $\mathscr{N} \vDash R_{i, x}^{j}(\bar{y}) \leftrightarrow \varphi_{i}^{j}(x, \bar{y})$.

Let us assume that in some definable way there is an enumeration of all $\mathscr{N}$-finite sequences of elements of $N$. We denote by (a) the sequence enumerated by a, and denote the length of (a) by $\ell(a)$. We say $(a) \triangleleft(b)$ iff $\ell(a)=\ell(b)$ and whenever $c<\ell(a)$, then $(a)_{c} \triangleleft(b)_{c}$.

Then for each $n<\omega$ it is easily seen that the following is a theorem of $\boldsymbol{P}$ :

$\left(*_{n}\right)$ For every sequence $(a)$ there is $(b) D(a)$ such that for all $i, j<n$, whenever $x<\ell(a), R_{i, x}^{j}$ is dense and $c_{0}, \cdots, c_{j-1}<\ell(a)$, then $\mathscr{N} \vDash R_{i, x}^{j}\left((b)_{c_{0}}, \cdots,(b)_{c_{j-1}}\right)$.

Now let $\left\langle c_{n}: n\langle\omega\rangle\right.$ be an increasing cofinal sequence in $\mathscr{N}$. By induction we get, for each $n<\omega$, sequences $\left(a_{n}\right)$ and $\left(b_{n}\right)$ where $\iota\left(a_{n}\right)=\ell\left(b_{n}\right)=c_{n}$. Let $\left(a_{0}\right)$ be the identity sequence of length $c_{0}$. Now suppose that we have $\left(a_{n}\right)$. In $\left(*_{n}\right)$ take $(a)$ to be $\left(a_{n}\right)$ and set $\left(b_{n}\right)=(b)$. Now let $\left(a_{n+1}\right)$ be the sequence of length $c_{n+1}$ such that $\left(a_{n+1}\right) c=\left(b_{n}\right)_{c}$ when $c<c_{n}$, and $\left(a_{n+1}\right)_{c}=c$ when $c_{n} \leqq c<c_{n+1}$.

For each $c \in N$ there is $n<\omega$ such that $c_{n} \leqq c<c_{n+1}$. Let

$$
X(c)=\bigcup\left\{F\left(\left(a_{i}\right)_{c}\right): n<i<\omega\right\} .
$$

Clearly $c=\left(a_{n+1}\right)_{c} \triangleleft\left(a_{n+2}\right)_{c} \triangleleft \cdots$, so that $F(c)$ is an initial segment of $X(c)$. It is clear, because of sentences $\left(*_{n}\right)$, that $\mathscr{X}=\{X(c): c \in N\}$ is generic.

In Theorem 2.7 suppose that card $(N)=\kappa$. Then it is possible to get such an $\mathscr{Q}$ for which each $\mathscr{N}$-finite set is an initial segment of $\kappa^{\omega}$ different $X \in \mathscr{Z}$. This is the best to expect since there are only $\kappa^{\omega}$ distinct classes.

In summary, let $\mathscr{N}$ be a model of cardinality $<\kappa$. We know that it has a $\kappa$-like elementary end-extension $\mathscr{M}$, and, if $\kappa$ is regular 
then in any such $\kappa$-like $\mathscr{C}$ the collection of all classes satisfies replacement; however, according to Theorem 1.5 there is even such an $\mathscr{C l}$ in which all the classes are definable. More generally, if $c f(\kappa)>\omega$ then by Theorem 1.6 we can choose $\mathscr{C}$ so that each class satisfying replacement is definable, and thus $\mathscr{C}$ has no generic classes. This is in contrast to the case when $c f(\kappa)=\omega$; then Theorem 2.7 tells us that $\mathscr{C}$ must have many generic classes. In the next section we show that, even if $c f(\kappa)>\omega$, we can get a $\kappa$ like $\mathscr{C l}$ with many generic classes.

3. Extending generic classes. We show in this section how to construct elementary end-extensions which extend generic classes. Iterations of these extensions result in $\kappa$-like models which have many classes. The next theorem generalizes the MacDowell-Specker Theorem and Lemma 1.4.

Theorem 3.1. If $\mathscr{P}$ is $\mathscr{N}$-generic, then there is a proper

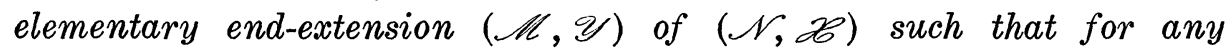
class $Y$ of $\mathscr{K}, Y \cap N$ is definable in $(\mathscr{N}, \not{Z})$.

Proof. We first describe the plan of attack. By Lemma 2.5 we know that the model $(\mathscr{N}, \mathscr{Z})$ satisfies induction. Thus, for each finite subset $\mathscr{Y}$ of $\mathscr{X}$ there is a proper elementary end-extension of $(\mathscr{N} ; \mathscr{Y})$. However, we want to construct these extensions in some uniform way so that they all cohere. That is, we want to be able to form their direct limit using Tarski's Union Theorem (Theorem 1.9 of [5]) so as to get a proper elementary end-extension of $(\mathscr{N} ; \mathscr{X})$.

We use the following piece of notation. If $e: n \rightarrow j$ and $\bar{a}=$ $\left\langle a_{0}, \cdots, a_{j-1}\right\rangle$, then we set $P_{e}(\bar{a})=\bar{b}$, where $b_{k}=a_{e(k)}$ for each $k<n$. If $R$ is a set of $j$-tuples, then we also set $P_{e}(R)=\left\{P_{e}(\bar{a}): \bar{a} \in R\right\}$. Our aim is to form a directed system $\langle\mathscr{L}(\bar{X}): \bar{X} \in D\rangle$, where $D$ is the set of all finite sequences of distinct elements of $\mathscr{Z}$, and $\bar{X} \prec \bar{Y}$ whenever there is some (necessarily unique) function $e: n \rightarrow j$ such that $P_{e}(\bar{Y})=\bar{X}$. Each $\mathscr{C}(\bar{X})$ is to be an elementary end-extension of $\mathscr{N}$ such that each $X_{i}$ is an initial segment of an $\mathscr{C}(\bar{Y})$-finite set. Furthermore, whenever $\bar{X} \prec \bar{Y}$, there is an elementary embedding $f_{\bar{X}, \bar{Y}}: \mathscr{C}(\bar{X}) \rightarrow \mathscr{C}(\bar{Y})$ which fixes the elements of $\mathscr{N}$; and if $\bar{X} \prec \bar{Y} \prec \bar{Z}$, then $f_{\bar{X}, \bar{Z}}=f_{\bar{Y}, \bar{Z}} \circ f_{\bar{X}, \bar{Y}}$. Then the direct limit of $\langle\mathscr{C}(\bar{X}): \bar{X} \in D\rangle$ is the desired model. As in the proof of Theorem 1.1, the models $\mathscr{C}(\bar{X})$ are constructed by getting a certain ultrafilter $V(\bar{X})$, and then letting $\mathscr{C}(\bar{X})$ be the Skolem ultrapower $\mathscr{N}^{v(\bar{X})}$. The problem of the coherence of all the $\mathscr{C}(\bar{X})$ is then transferred to that of the coherence of all the ultrafilters $V(\bar{X})$. 
To proceed with the proof, let $\left\langle\varphi_{n}\left(t, x, y_{0}, \cdots, y_{n-1}\right): n\langle\omega\rangle\right.$ be a list of all formulas with the free variables $t, x, y_{0}, y_{1}, \cdots$. Our first goal is to get formulas $\psi_{n}(z, s)$ with certain properties. Think of $\psi_{n}(z, s)$ in the following way: For each $n<\omega$ and each $a \in N$, let $S_{a}^{n}$ be the set of $\mathscr{N}$-finite sequences $p$ such that $p=(s)$ for some $s$ for which $\psi_{n}(a, s)$ holds in $\mathscr{N}_{\text {. }}$ (Recall that $(s)$ denotes the $\mathscr{N}$ finite sequence enumerated by $s$.) Then the sets $S_{a}^{n}$ should have the following properties (all of which can be formulated as theorems of $P$ ):

(1) $S_{a}^{n}$ is a nonempty set of sequences each of length $a$.

(2) If $p \in S_{a}^{n}$ and $b<a$, then $b \triangleleft p_{b}$.

(3) If $p \in S_{a}^{n}$ and $p \triangleleft q$, then there exists $r \in S_{a}^{n}$ such that $q \triangleleft r$ (that is, $S_{a}^{n}$ is dense in itself).

(4) Suppose that $p \in S_{b}^{n}$, and that $f$ is a definable function such that $c \leqq f(c)<b$ for each $c<a$. Then $q \in S_{a}^{n}$ where $q_{c}=p_{f(c)}$ for each $c<a$.

(5) For each $n<\omega$ and each $t$, let $f_{t}^{n}$ be the (definable) $n$-ary function such that $f_{t}^{n}\left(y_{0}, \cdots, y_{n-1}\right)$ is the least $x$ for which $\varphi_{n}(t, x, \bar{y}) \vee$ $x=t$ holds in $\mathscr{N}$. Whenever $t<a$ and $a_{0}<\cdots a_{n-1}<a$, then there is $b$ such that for any $p \in S_{a}^{n}, f_{t}^{n}\left(p_{a_{0}}, \cdots, p_{a_{n-1}}\right)=b$.

(6) $S_{a}^{m} \subset S_{a}^{n}$ whenever $n<m<\omega$.

To show the existence of the formulas $\psi_{n}(z, s)$ satisfying properties (1)-(6), one uses induction on $n$, and then uses induction on the variable $z$ inside of the model. The essence of the formal induction is the following lemma.

Lemma. For each $n$ let $T_{n}$ be a set of finite sequences all of the same length such that $T_{n}$ is dense in itself. Furthermore, suppose that $T_{m} \supset T_{n}$ whenever $m<n$, and that $P$ is a finite partition of $T_{0}$. Then there is $T \subset T_{0}$ such that $T \subset E$ for some $E \in P$, and for each $n, T \cap T_{n}$ is dense in itself.

This lemma is easily proved. In fact it can easily be formulated and proved as a theorem scheme of $\boldsymbol{P}$. Using this lemma we prove the existence of sets $S_{a}^{m}$. This proof can of course be given in $\boldsymbol{P}$ so that we actually show the existence of formulas $\psi_{m}(z, s)$.

Let us suppose that $\varphi_{0}(t, x)$ is a universally valid formula so that we can set

$$
\begin{aligned}
& S_{a}^{0}=\left\{p: p \text { is a sequence of length } a \text { such that } b \triangleleft p_{b}\right. \\
& \quad \text { for each } b<a\} .
\end{aligned}
$$

Also set $S_{0}^{m}=\{\varnothing\}$ for each $m<\omega$. As an inductive hypothesis, suppose that $S_{x}^{m}$ has been defined for each $m \leqq n$ and each $x$, and 
that $S_{0}^{n+1}, \cdots, S_{a}^{n+1}$ have also been defined. We define $S_{a+1}^{n+1}$. For every $c>a$, let $T_{c}$ be the set of sequences $p$ such that $p=q \mid(a+1)$ for some $q \in S_{c}^{n}$ and $p \mid a \in S_{a}^{n+1}$. Each $T_{c}$ is dense in itself, and $c<d$ implies $T_{\mathrm{c}} \supset T_{d}$. Partition $T_{a+1}$ as follows: If $p, q \in T_{a+1}$, then $p$ is equivalent to $q$ iff whenever $m \leqq n+1, t<a+1$ and $a_{0}<\cdots<a_{m-1}<$ $a+1$, then $f_{t}^{m}\left(p_{a_{0}}, \cdots, p_{a_{m-1}}\right)=f_{t}^{m}\left(q_{a_{0}}, \cdots, q_{a_{m-1}}\right)$. This partition is clearly $\mathscr{N}$-finite. The hypotheses of the lemma are now met. Hence, there is $T \subset T_{a+1}$ such that $T \subset E$ for some $E \in P$ and, for each $c>a$, $T \cap T_{c}$ is dense in itself. Let $S_{a+1}^{n+1}=T$. One easily checks that the $S_{a}^{m}$ so defined satisfy the properties (1)-(6).

Next, we give the construction of the model $\mathscr{M}$. Let $\bar{x}=$ $\left\langle x_{0}, \cdots, x_{n}\right\rangle$ be an $(n+1)$-tuple of elements of $N$. For each $a \in N$ and $m<\omega$, define

$$
\begin{aligned}
R_{a}^{m}(\bar{x}) & =\left\{\left\langle p_{x_{0}}, \cdots, p_{x_{n}}\right\rangle: p \in S_{a}^{m}\right\}, \\
U^{m}(\bar{x}) & =\left\{R \subset N^{n+1}: R \text { is definable and } R \supset R_{a}^{m}(\bar{x}) \text { for some } a \in N\right\}, \\
U(\bar{x}) & =\bigcup\left\{U^{m}(\bar{x}): m<\omega\right\} .
\end{aligned}
$$

The following properties hold:

(1*) $U(\bar{x}) \neq \varnothing$.

$\left(2^{*}\right)$ If $S \supset R \in U(\bar{x})$ and $S \in N^{n+1}$ is definable, then $S \in U(\bar{x})$.

$\left(3^{*}\right)$ If $R, S \in U(\bar{x})$, then $R \cap S \in U(\bar{x})$.

$\left(4^{*}\right)$ If $\bar{x} \triangleleft \bar{y}$, then $U(\bar{x}) \subset U(\bar{y})$.

$\left(5^{*}\right) \quad\left\{\bar{y} \in N^{n+1}: \bar{x} \triangleleft \bar{y}\right\} \in U(\bar{x})$.

$\left(6^{*}\right)$ If $R \subset N^{n+1}$ is definable, then for some $m<\omega$ the set

$$
\left\{\bar{x} \in N^{n+1}: R \in U^{m}(\bar{x}) \text { or }\left(N^{n+1}-R\right) \in U^{m}(\bar{x})\right\}
$$

is dense. More generally, if $f: N^{n+1} \rightarrow N$ is definable with bounded range, then for some $m$ the set $\left\{\bar{x} \in N^{n+1}: f^{-1}(b) \in U^{m}(\bar{x})\right.$ for some $b \in N\}$ is dense.

Property $\left(1^{*}\right)$ follows immediately from (1), property $\left(2^{*}\right)$ is trivial, and $\left(3^{*}\right)$ can easily be proved from (4) and (6). Property $\left(5^{*}\right)$ is immediate from (2), and $\left(6^{*}\right)$ is a consequence of (5). Property $\left(4^{*}\right)$ follows from (4). For, suppose that $\bar{x} \triangleleft \bar{y}$ and $R \in U(\bar{x})$. Then $R=R_{a}^{m}(\bar{x})$ for some $m$ and $a$. Let $b$ be sufficiently large: choose $b>\max \left(y_{0}, \cdots, y_{n}, a\right)$. Now let $f$ be the definable function such that

$$
\begin{aligned}
f\left(x_{i}\right) & =y_{i} \text { for } i \leqq n, \\
f(x) & =x \text { otherwise } .
\end{aligned}
$$

Then using (4) we see that $R_{b}^{m}(\bar{y}) \subset R_{a}^{m}(\bar{x})$. Hence $R \in U(\bar{y})$, so that $\left(4^{*}\right)$ holds.

Now let $\bar{X}=\left\langle X_{0}, \cdots, X_{n-1}\right\rangle$ be a sequence of distinct classes of N. Define 


$$
V(\bar{X})=\left\{R \subset N^{n}: R \in U(\bar{x}) \text { and } \bar{x} \in \bar{X}\right\} .
$$

Properties $\left(1^{*}\right)-\left(4^{*}\right)$ imply that $V(\bar{X})$ is a filter in the Boolean algebra of definable $n$-ary relations. $\left(5^{*}\right)$ implies that this filter is nonprincipal. Suppose, furthermore, that each $X_{i}$ is a member of the generic collection $\mathscr{R}$. Hence it follows from $\left(6^{*}\right)$ (more accurately, from the formalization of $\left(6^{*}\right)$ as a theorem scheme of $\boldsymbol{P}$ ) that $V(\bar{X})$ is an ultrafilter. More generally, $\left(6^{*}\right)$ implies that some member of each $\mathscr{N}$-finite collection whose union is $N^{n}$ is a member of $V(\bar{X})$.

We can now form the Skolem ultrapower $\mathscr{C}(\bar{X})=\mathscr{N}^{v(\bar{X})}$ of $\mathscr{N}$. Since $V(\bar{X})$ is closed under $\mathscr{N}$-finite intersections, the model $\mathscr{C}(\bar{X})$ is an elementary end-extension of $\mathscr{r}$. We wish to show that each $X_{i}$ is an initial segment of an $\mathscr{C}(\bar{X})$-finite set. It suffices to show that there is $f \in \mathscr{l}(\bar{X})$ such that for all $a \in X_{i}, \quad \mathscr{C}(\bar{X}) \vDash a \triangleleft f$. By $\left(5^{*}\right)$ the function $f$ defined by $f(\bar{a})=a_{i}$ clearly has this property.

The ultrafilters $V(\bar{X})$ have a certain property which guarantees that the models $\mathscr{C}(\bar{X})$ cohere. Suppose that $e: n \rightarrow j, \bar{Y} \in \mathscr{Z}^{j}$ and $\bar{X}=P_{e}(\bar{Y}) \in \mathscr{X}^{n}$. We easily get from (4) that

$$
V(\bar{X})=\left\{P_{e}(R): R \in V(\bar{Y})\right\} \text { • }
$$

Thus there is an elementary embedding $f_{\bar{X}, \bar{Y}}: \mathscr{C l}(\bar{X}) \rightarrow \mathscr{C l}(\bar{Y})$ which fixes the elements of $\mathscr{N}$. If $g: N^{n} \rightarrow N$ is definable in $\mathscr{N}$, then let

$$
f_{X, \bar{Y}}(g)=g \circ P_{e} \text {. }
$$

By the use of Łoś' Theorem for Skolem ultrapowers, it is easily verified that $f_{\bar{X}, \bar{Y}}$ is an elementary embedding.

We form a directed system of structures. Recall that $D$ is the set of finite sequences of distinct elements of $\mathscr{R}$. Thus $(D, \prec)$ is a directed set such that whenever $\bar{X} \prec \bar{Y}$, then $f_{\bar{X}, \bar{Y}}: \angle \mathscr{C}(\bar{X}) \rightarrow \mathscr{C l}(\bar{Y})$ is an elementary embedding. Also it is clear that if $\bar{X} \prec \bar{Y} \prec \bar{Z}$, then $f_{\bar{X}, \bar{Z}}=f_{\bar{Y}, \bar{Z}} \circ f_{X, Y}$. Thus, $\langle\mathscr{C}(\bar{X}): \bar{X} \in D\rangle$ is a directed system of structures, so that now applying Tarski's Union Theorem we get the direct limit $\mathscr{L}_{0}$, which is an elementary end-extension of $\mathscr{N}$; and each $X \in \mathscr{X}$ is an initial segment of some $\mathscr{L}_{0}$-finite set.

We want, however, a proper elementary end-extension of $(\mathcal{H}, \mathbb{Z})$. To this end, let $\left\langle\mathscr{C}_{i}: i \leqq \omega\right\rangle$ be a MacDowell-Specker chain, and set $\mathscr{l}=\mathscr{C}_{\omega}$. Since each $X \in \mathscr{C}$ is an initial segment of an $\mathscr{C l}$-finite set and $c f(\mathscr{C})=\omega$, then according to Theorem 2.7 there is an $\mathscr{C}$ generic $\mathscr{Y}$ such that $(\mathscr{N}, \mathscr{X}) \subset(\mathscr{M}, \mathscr{Y})$. But then $(\mathscr{N}, \mathscr{Z}) \prec(\mathscr{K}, \mathscr{Y})$ by Lemma 2.6 .

Finally we must show that for any class $Y$ of $\mathscr{C}, Y \cap N$ is definable in $(\mathscr{N}, \mathscr{X})$. Let $Y$ be a class of $\mathscr{l l}$. From Lemmas 1.3 
and 1.4 it is clear that $Y \cap M_{0}$ is definable in $\mathscr{A}_{0}$. We now proceed as in the proof of Lemma 1.4. Let $b \in\left(Y \cap M_{0}\right)-N$. Then there is $c \in M$ such that $F(c)=\{x \in Y: \mathrm{x}<b\}$. Let $\bar{X} \in D$ be such that $c \in \mathscr{l}(\bar{X})$. Then, in the construction of $\mathscr{L}(\bar{X})$, the element $c$ corresponds to a function definable in $\mathscr{N}$. Now the ultrafilter $V(\bar{X})$ is definable in $(\mathscr{N}, \bar{X})$; hence, it is clear that $F(c) \cap N=Y \cap N$ is definable in $(\mathscr{N}, \bar{X})$.

We use Theorem 3.1 along with the existence of certain trees to build models with special properties. The method is based on [3].

We say $(T,<)$ is a tree iff $<$ is a partial ordering of $T$ such that the set of predecessors of any element is well-ordered. We let $T_{\alpha}$ be the set of elements whose set of predecessors has order type $\alpha$. A tree $(T,<)$ is a $\kappa$-tree iff both (i) $T_{\alpha}=\varnothing$ iff $\kappa \leqq \alpha$, and (ii) for $a<\kappa$, card $\left(\bigcup\left\{T_{\nu}: \nu<\alpha\right\}\right)<\kappa$. A branch of a tree is a maximal, linearly ordered subset of the tree. We consider $\kappa$-trees which have exactly $\lambda$ branches of length $\kappa$. Such a tree with $\lambda>\kappa$ is a $\kappa$-Kurepa tree. It is known that under the assumption of Gödel's Axiom of Constructibility $V=L$, there exist $\kappa$-Kurepa trees for each $\kappa \geqq \omega$. (See [2].) Notice that whenever $1 \leqq \lambda \leqq \kappa$, there are trivial $\kappa$-trees with exactly $\lambda$ branches of length $\kappa$.

Suppose that $\lambda=0,1$ or $\lambda$ is an infinite cardinal. We say that $\mathscr{N}$ has generic dimension $\lambda$ iff there is an $\mathscr{N}$-generic $\mathscr{Z}$ such that each class of $\mathscr{V}$ is definable in $(\mathscr{N}, \mathscr{Z})$ and card $(\mathscr{Z})=\lambda$. The generic dimension, if it exists, is unique. For, suppose that $\mathscr{E}$ and $\mathscr{Y}$ are $\mathscr{N}$-generic such that each class of $\mathscr{N}$ is definable in $(\mathscr{N}, \mathscr{Z}$ ) and $(\mathscr{N}, \mathscr{Y})$. If $\omega \leqq \operatorname{card}(\mathscr{X}) \leqq \operatorname{card}(\mathscr{Y})$, then there is $\mathscr{Y}_{0} \subset \mathscr{Y}$ such that card $(\mathscr{Z})=\operatorname{card}\left(\mathscr{Z}_{0}\right)$ and each $X \in \mathscr{Z}$ is definable in $\left(\mathscr{N}, \mathscr{V}_{0}\right)$, so that each $Y \in \mathscr{Y}$ is definable in $\left(\mathscr{N}, \mathscr{Y}_{0}\right)$. Hence $\mathscr{Y} \mathscr{V}_{0}=\mathscr{Y}$ and so card $(\mathscr{Z})=$ card $(\mathscr{Y})$. Argue similarly for finite generic dimension. Theorem 1.5 asserts the existence of $\kappa$-like models with generic dimension 0 for each regular $\kappa>\omega$; the next theorem generalizes this assertion. Notice that if $\lambda \geqq \kappa$ and $\mathscr{N}$ is $\kappa$-like with generic dimension $\lambda$, then $\mathscr{T}$ has exactly $\lambda$ classes.

THEOREM 3.2. Suppose that $\kappa$ is regular, $\lambda \geqq \omega$ or $\lambda=1$, and that there is a $\kappa$-tree with exactly $\lambda$ branches of length $\kappa$. Then for each model $\mathscr{N}$ of cardinality $<\kappa$ there is a $\kappa$-like elementary endextension $/ \mathrm{ll}$ with generic dimension $\lambda$.

Proof. Let $(T,<)$ be a $\kappa$-tree with exactly $\lambda$ branches of length $\kappa$. Without loss of generality we can assume that each element of $T$ is contained in some branch of length $\kappa$. By induction we get $\left\langle\mathscr{N}_{2}: \nu\langle\kappa\rangle\right.$ and $\left\langle X_{t}: t \in T\right\rangle$ as follows. 
Suppose $\nu=0$. Let $\alpha=\operatorname{card}\left(T_{0}\right) \cdot \omega$ (ordinal multiplication). Let $\left\langle\mathscr{M}_{\xi}: \xi \leqq \alpha\right\rangle$ be a MacDowell-Specker chain such that $\mathscr{A}_{0}=\mathscr{N}$. We set $\mathscr{N}_{0}=\mathscr{A}_{\alpha}$. According to Theorem 2.7 there is an $\mathscr{N}_{0}$-generic $\mathscr{X}$ such that card $(\mathscr{Q})=\operatorname{card}\left(T_{0}\right)$. So we let $\left\{X_{t}: t \in T_{0}\right\}$ be $\mathscr{N}_{0}$-generic, and $X_{s}$ and $X_{t}$ are distinct whenever $s$ and $t$ are.

Now suppose $\nu<\kappa$ and that we have $\mathscr{N}_{\nu}$ and $\left\{X_{t}: t \in T_{\nu}\right\}$ which is $\mathscr{N}_{\nu}$-generic, and $X_{s}$ and that $X_{t}$ are distinct whenever $s$ and $t$ are. We use Theorem 3.1 with $\mathscr{N}=\mathscr{N}_{\nu}$ and $\mathscr{X}=\left\{X_{t}: t \in T_{\nu}\right\}$ to get $\mathscr{M}$ as in the theorem. Furthermore, we can get $\mathscr{C l}$ such that $c f(\mathscr{C})=\omega$ and $\operatorname{card}(M)=\operatorname{card}\left(N_{\nu}\right)+\operatorname{card}\left(T_{\nu}\right)$. Set $\mathscr{N}_{\nu+1}=$ M. Now using Theorem 2.7 again we can find $\mathscr{M}_{2+1}$-generic $\mathscr{Z}$ such that card $(\mathscr{P})=\operatorname{card}\left(T_{\nu+1}\right)$. Actually we can get $\left\{X_{t}: t \in T_{\nu+1}\right\}$ which, besides having distinct elements and being $\mathscr{N}_{\nu+1}$-generic, has the property that whenever $s \in T_{\nu}, t \in T_{\nu+1}, s<t$, then $X_{s}$ is a proper initial segment of $X_{t}$.

For the case of limit ordinals, let $\delta<\kappa$ be a limit ordinal. Then let $\mathscr{N}_{\delta}=\bigcup\left\{\mathscr{N}_{\alpha}: \alpha<\delta\right\}$, and for each $t \in T_{\delta}$, let $X_{t}=\bigcup\left\{X_{s}: s<t\right\}$. By Lemma $2.4\left\{X_{t}: t \in T_{\delta}\right\}$ is $\mathscr{N}_{\delta}$-generic.

Now let $\mathscr{C}=\bigcup\left\{\mathscr{N}_{\nu}: \nu<\kappa\right\}$. We claim that $\mathscr{A}$ has the desired properties. Clearly $\mathscr{C}$ is a $\kappa$-like elementary end-extension of $\mathscr{N}$. Now let

$$
\mathscr{Y}=\left\{\bigcup\left\{X_{t}: t \in B\right\}: B \text { is a branch of length } \kappa\right\} \text {. }
$$

By the construction, card $(\mathscr{Y})=\lambda$. We continue as in the proof of Theorem 1.5. Let $X$ be a class of $\mathscr{A}$. By constructing an elementary tower of models, we easily see that there is a $\nu<\kappa$ and a sequence $\left\langle Y_{t}: t \in T_{\nu}\right\rangle$ of elements of $\mathscr{Y}$ such that

$$
\left(\mathscr{N}_{\nu}, X \cap \mathscr{N}_{\nu}, X_{t}\right)_{t \in T_{\nu}} \prec\left(\mathscr{L}, X, Y_{t}\right)_{t \in T_{\nu}} .
$$

By Lemma 1.3, $X \cap N_{\nu+1}$ is a class of $\mathscr{N}_{\nu+1}$ so that $X \cap N_{\nu}$ is definable in $\left(\mathscr{N}_{\nu}, X_{t}\right)_{t \in T_{\nu}}$. But then $X$ is definable in $(\mathscr{M}, \mathscr{Y})$.

Theorem 3.2 is the best possible in the following sense: if $\kappa$ is regular, $\lambda \geqq \omega$, and there is a $\kappa$-like model $\mathscr{N}$ with generic dimension $\lambda$, then there is a $\kappa$-tree with exactly $\lambda$ branches. We need only consider the case that $\lambda>\kappa$. Let $\mathscr{N}$ be such a $\kappa$-like model and let $\mathscr{Z}$ be the collection of all classes of $\mathscr{N}$. Now choose $C \subset N$ to have order type $\kappa$. Let $T=\{(c, A): c \in C$ and $A=\{x \in X: x<c\}$ for some $X \in \mathscr{Z}\}$, and let $(c, A)<(d, B)$ iff $c<d$ and $A=\{x \in B: x<c\}$. Then $(T,<)$ is the desired $\kappa$-tree, each branch of length $\kappa$ corresponding to a unique element of $\mathscr{X}$.

The next theorems can be proved with a construction like that in the proof of Theorem 3.2. 
THEOREM 3.3. Suppose that there is a $\kappa$-tree with at least $\lambda$ branches of length $\kappa$. Then for each model $\mathscr{N}$ of cardinality $<\kappa$ there is a $\kappa$-like elementary end-extension $\mathscr{C l}$ and an $\mathscr{L}$-generic $\mathscr{Y}$ of cardinality $\lambda$.

THEOREM 3.4. Suppose that $c f(\kappa)>\omega$ and that there is a $\kappa$-tree with exactly $\lambda$ branches of length $\kappa$. Then for each model $\mathscr{N}$ of cardinality $<\kappa$ there is a $\kappa$-like elementary end-entension $\mathscr{C l}$ and an $\mathscr{C l}$-generic $\mathscr{Y}$ of cardinality $\lambda$ such that each class of $\mathscr{A l}$ which satisfies replacement is definable in $(\mathscr{M}, \mathscr{Y})$.

If we do not require that the models we get be $\kappa$-like but merely that they have cardinality $\kappa$, then we can do better. Let $D(\kappa)$ be the least cardinal $\lambda$ such that every tree with at most $\kappa$ nodes has fewer than $\lambda$ branches having length the length of the tree. It is easily shown that $\kappa^{+}<D(\kappa)$ for all $\kappa$. The following theorem can be proved by the same construction as in Theorem 3.2.

THEOREM 3.5. For each model $\mathscr{N}$ such that $\operatorname{card}(N) \leqq \kappa$ and $\lambda<D(\kappa)$, there is an elementary end-extension $\mathscr{C}$ and an $\mathscr{C}$ generic $\mathscr{Y}$ of cardinality $\lambda$.

Theorem 3.5 is the best possible for, in general, if $\mathscr{X}$ is $\mathscr{N}$ generic, then card $(\mathscr{X})<D(\operatorname{card}(N))$.

We can apply these results to models of $Z F$ set theory. For models of $Z F$ a generic collection of classes is simply a collection of mutually Cohen-generic reals. The theory $Z F$ does not, however, fall into the general scheme of Peano arithmetic described at the beginning of the Introduction. But this is easily remedied by collapsing the universe onto $\omega$. In a model $\mathscr{C l}$ of $Z F$, consider the notion of forcing in which the forcing conditions are one-to-one functions with natural number domains and which are ordered by inclusion. If $G$ is a generic filter, then $F=\bigcup G$ is a one-to-one function from $\omega$ onto $M$. Then the structure $(\mathscr{C}, F)$ still satisfies (finite) induction and can be considered (essentially) as a model of some theory $\boldsymbol{P}$. Since generic filters always exist in countable models, we get the following theorems:

THEOREM 3.6. Suppose that there is a $\kappa$-tree with at least $\lambda$ branches of length $\kappa$. Then any model of $Z F$ of cardinality $<\kappa$ has an elementary extension $\mathscr{L}$ such that

(i) each $\mathscr{L}$-finite set has cardinality $<\kappa$;

(ii) there are exactly $\kappa$ natural numbers in $\mathscr{C}$;

(iii) th has $\lambda$ mutually Cohen-generic reals. 
THEOREM 3.7. If $\lambda<D(\kappa)$, then any model of $Z F$ of cardinality $\leqq \kappa$ has an elementary extension of cardinality $\kappa$ which has $\lambda$ mutually Cohen-generic reals.

\section{REFERENCES}

1. T. Frayne, A. C. Morel, and D. Scott, Reduced direct products, Fund. Math., 51 (1962), 50-82.

2. R. B. Jensen, Some Combinatorial Properties of $L$ and $V$, (mimeographed notes, 1970).

3. H. J. Keisler, A Short Course in Model Theory, (mimeographed notes prepared by

D. Guaspari), Cambridge, 1971.

4. R. MacDowell, and E. Specker, Modelle der Arithmetik, Infinitistic Methods, Proceedings of the Symposium on Foundations of Mathematics, Warsaw 1959, New-YorkOxford-London-Paris and Warsaw, 1961, 257-263.

5. J. R. Shoenfield, Unramified forcing, Axiomatic Set Theory, Proceedings of Symposia in Pure Mathematics, Vol. XIII, Part 1, American Mathematical Society, Providence, Rhode Island, (1971), 357-381.

6. T. Skolem, Über die Nicht-Charakterisierbarkeit der Zahlenreihe mittels endlich oder abzähler unendlich vieler Aussagen mit ausschliesslich Zahlenvariablen, Fund. Math., 23 (1934), 150-161.

7. A. Tarski and R. L. Vaught, Arithmetical extensions of relational systems, Compositio Math., 13 (1957), 81-102.

Received December 22, 1971 and in revised form July 10, 1972.

YALE UNIVERSITY 


\section{PACIFIC JOURNAL OF MATHEMATICS}

\section{EDITORS}

D. Gilbarg and J. Milgram

Stanford University

Stanford, California 94305

\author{
R. A. Beaumont \\ University of Washington \\ Seattle, Washington 98105
}

J. DuGUNDJI

Department of Mathematics

University of Southern California

Los Angeles, California 90007

Richard ARENS

University of California

Los Angeles, California 90024

\section{ASSOCIATE EDITORS}
E. F. BECKENBACH
B. H. NeumanN
F. WOLF
K. YosHIDA

\section{SUPPORTING INSTITUTIONS}

UNIVERSITY OF BRITISH COLUMBIA

CALIFORNIA INSTITUTE OF TECHNOLOGY

UNIVERSITY OF CALIFORNIA

MONTANA STATE UNIVERSITY

UNIVERSITY OF NEVADA

NEW MEXICO STATE UNIVERSITY

OREGON STATE UNIVERSITY

UNIVERSITY OF OREGON

OSAKA UNIVERSITY

\author{
UNIVERSITY OF SOUTHERN CALIFORNIA \\ STANFORD UNIVERSITY \\ UNIVERSITY OF TOKYO \\ UNIVERSITY OF UTAH \\ WASHINGTON STATE UNIVERSITY \\ UNIVERSITY OF WASHINGTON
* * * *
AMERICAN MATHEMATICAL SOCIETY \\ NAVAL WEAPONS CENTER
}

The Supporting Institutions listed above contribute to the cost of publication of this Journal, but they are not owners or publishers and have no responsibility for its content or policies.

Mathematical papers intended for publication in the Pacific Journal of Mathematics should be in typed form or offset-reproduced, (not dittoed), double spaced with large margins. Underline Greek letters in red, German in green, and script in blue. The first paragraph or two must be capable of being used separately as a synopsis of the entire paper. The editorial "we" must not be used in the synopsis, and items of the bibliography should not be cited there unless absolutely necessary, in which case they must be identified by author and Journal, rather than by item number. Manuscripts, in duplicate if possible, may be sent to any one of the four editors. Please classify according to the scheme of Math. Rev. Index to Vol. 39. All other communications to the editors should be addressed to the managing editor, Richard Arens, University of California, Los Angeles, California, 90024.

50 reprints are provided free for each article; additional copies may be obtained at cost in multiples of 50 .

The Pacific Journal of Mathematics is issued monthly as of January 1966. Regular subscription rate: $\$ 48.00$ a year (6 Vols., 12 issues). Special rate: $\$ 24.00$ a year to individual members of supporting institutions.

Subscriptions, orders for back numbers, and changes of address should be sent to Pacific Journal of Mathematics, 103 Highland Boulevard, Berkeley, California, 94708.

PUBLISHED BY PACIFIC JOURNAL OF MATHEMATICS, A NON-PROFIT CORPORATION

Printed at Kokusai Bunken Insatsusha (International Academic Printing Co., Ltd.), 270, 3-chome Totsuka-cho, Shinjuku-ku, Tokyo 160, Japan. 


\section{Pacific Journal of Mathematics}

\section{Vol. 46, No. 2 December, 1973}

Christopher Allday, Rational Whitehead products and a spectral sequence of

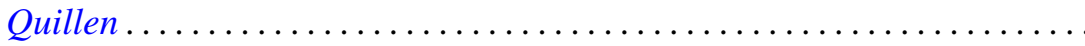

James Edward Arnold, Jr., Attaching Hurewicz fibrations with fiber

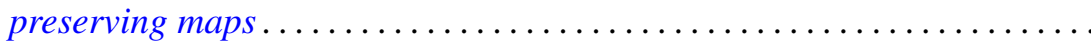

Catherine Bandle and Moshe Marcus, Radial averaging transformations with various metrics.................................

David Wilmot Barnette, A proof of the lower bound conjecture for convex

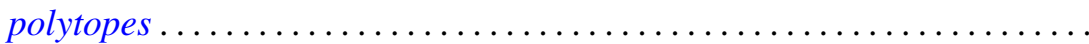

Louis Harvey Blake, Simple extensions of measures and the preservation of

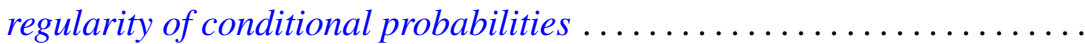

James W. Cannon, New proofs of Bing's approximation theorems for

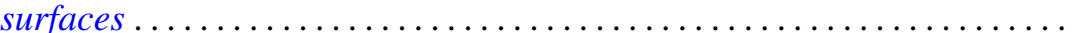

C. D. Feustel and Robert John Gregorac, On realizing HNN groups in

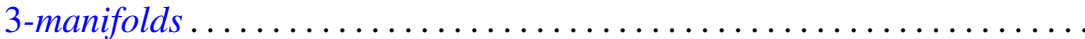

Theodore William Gamelin, Iversen's theorem and fiber algebras . . . . . . . . 389

Daniel H. Gottlieb, The total space of universal fibrations . . . . . . . . . . . .

Yoshimitsu Hasegawa, Integrability theorems for power series expansions of

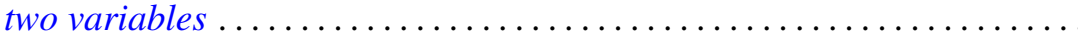

Dean Robert Hickerson, Length of period simple continued fraction expansion of $\sqrt{ } d$

Herbert Meyer Kamowitz, The spectra of endomorphisms of the disc algebra.

Dong S. Kim, Boundedly holomorphic convex domains

Daniel Ralph Lewis, Integral operators on $\mathscr{L}_{p}$-spaces ...

John Eldon Mack, Fields of topological spaces . . . . . . . . .

V. B. Moscatelli, On a problem of completion in bornology

Ellen Elizabeth Reed, Proximity convergence structures. .

Ronald C. Rosier, Dual spaces of certain vector sequence spaces .

Robert A. Rubin, Absolutely torsion-free rings

Leo Sario and Cecilia Wang, Radial quasiharmonic functions . .

James Henry Schmerl, Peano models with many generic classes .

H. J. Schmidt, The $\mathscr{F}$-depth of an $\mathscr{F}$-projector ............

Edward Silverman, Strong quasi-convexity. . . . . . . . . . . . . . . . . 549

Barry Simon, Uniform crossnorms ....................... 555

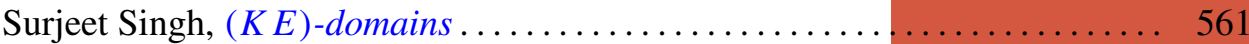

Ted Joe Suffridge, Starlike and convex maps in Banach spaces . . . . . . . . 575

Milton Don Ulmer, $C$-embedded $\Sigma$-spaces . . . . . . . . . . . . . . . . 591

Wolmer Vasconcelos, Conductor, projectivity and injectivity . . . . . . . . . 603 